\title{
A p53-derived apoptotic peptide derepresses p73 to cause tumor regression in vivo
}

\author{
Helen S. Bell, ${ }^{1}$ Christine Dufes, ${ }^{2}$ Jim O'Prey, ${ }^{1}$ Diane Crighton, ${ }^{1}$ Daniele Bergamaschi, ${ }^{3}$ Xin Lu, ${ }^{3}$ \\ Andreas G. Schätzlein, ${ }^{2}$ Karen H. Vousden, ${ }^{4}$ and Kevin M. Ryan'
}

${ }^{1}$ Tumour Cell Death Laboratory, Beatson Institute for Cancer Research, Glasgow, United Kingdom. ${ }^{2}$ Centre for Oncology and Applied Pharmacology, University of Glasgow, Glasgow, United Kingdom. ${ }^{3}$ Ludwig Institute for Cancer Research, University College London, London, United Kingdom. ${ }^{4}$ Tumour Suppression Laboratory, Beatson Institute for Cancer Research, Glasgow, United Kingdom.

\begin{abstract}
The tumor suppressor $\mathrm{p} 53$ is a potent inducer of tumor cell death, and strategies exist to exploit p53 for therapeutic gain. However, because about half of human cancers contain mutant $\mathrm{p} 53$, application of these strategies is restricted. $\mathrm{p} 53$ family members, in particular $\mathrm{p} 73$, are in many ways functional paralogs of $\mathrm{p53}$, but are rarely mutated in cancer. Methods for specific activation of $\mathrm{p} 73$, however, remain to be elucidated. We describe here a minimal p53-derived apoptotic peptide that induced death in multiple cell types regardless of p53 status. While unable to activate gene expression directly, this peptide retained the capacity to bind iASPP - a common negative regulator of $\mathrm{p} 53$ family members. Concordantly, in p53-null cells, this peptide derepressed $\mathrm{p} 73$, causing $\mathrm{p} 73$-mediated gene activation and death. Moreover, systemic nanoparticle delivery of a transgene expressing this peptide caused tumor regression in vivo via $\mathrm{p} 73$. This study therefore heralds what we believe to be the first strategy to directly and selectively activate $\mathrm{p} 73$ therapeutically and may lead to the development of broadly applicable agents for the treatment of malignant disease.
\end{abstract}

\section{Introduction}

The p53 tumor suppressor serves to protect against the proliferation of damaged cells that may otherwise go on to form a tumor (1). In response to various forms of cellular stress such as DNA damage, hypoxia, and oncogene activation, the levels of p53 accumulate initially at mitochondria and subsequently in the nucleus, where p53 mediates its tumor-suppressive effects through target gene activation (2). A number of tumor-suppressive consequences of p53 activation have been described, but it is the induction of cellcycle arrest, during which cellular damage can be repaired, and the induction of programmed cell death, which causes the eradication of damaged cells, that are considered the most important (3).

The type of response that follows p53 activation depends on a number of factors. Importantly, oncogenic transformation can cause a switch in the cell's response to p53 activation from growth arrest to programmed cell death (4). As a result, many tumor cells are more likely to undergo apoptosis following p53 activation than are corresponding normal cells (5), making the p53 pathway an excellent target for therapeutic intervention (6). A number of strategies have therefore been developed to directly and specifically target p53 $(7,8)$. However, the fact that approximately $50 \%$ of human cancers contain nonfunctional mutant p53 limits the application of these agents to specific tumor settings (9).

p63 and p73 are 2 p53-related proteins (10). p73 in particular (strictly the "TA" splice variants of $\mathrm{p} 73$, but hereafter referred to as p73 for simplicity) shares many functional characteristics with p53 and can induce cell death in multiple cell types $(11,12)$. However, although $\mathrm{p} 73$ has been shown to be important for chemosensitiv-

Nonstandard abbreviations used: ASPP, apoptosis stimulating protein of p53; HFF, human foreskin fibroblast; iASPP, inhibitory member of the ASPP family; MEF, mouse embryo fibroblast; PUMA, $p 53$ upregulated modulator of apoptosis; qPCR, quantitative real-time PCR; RPE, retinal pigment epithelial cell; shRNA, short hairpin RNA. Conflict of interest: The authors have declared that no conflict of interest exists. Citation for this article: J. Clin. Invest. 117:1008-1018 (2007). doi:10.1172/JCI28920. ity in tumor cells (13), in contrast to p53, it is rarely mutated in human cancer (14). Therefore, the development of agents that target p73 may result in therapeutic strategies that are more broadly applicable than those targeting p53. In this regard, we describe here the elucidation and use of a mechanism to directly activate p73 that causes p73-dependent cell death and tumor regression in the absence or presence of WT p53. The mechanism centers around the identification of a minimal apoptotic peptide from p53, which, while unable to directly cause p53 target gene activation, retains the ability to bind iASPP - a common inhibitor of 553 family proteins. By binding iASPP, this peptide disrupts the interaction between iASPP and p73 causing derepression of endogenous $\mathrm{p} 73$. This subsequently causes activation of $\mathrm{p} 73$ target genes and death. In line with these in vitro studies, administration of a transgene expressing this peptide in vivo caused tumor regression in a manner dependent on $\mathrm{p} 73$.

\section{Results}

A p53-derived 37-aa peptide induces cell death in multiple tumor cell types, but not in normal cells. A number of reports have indicated that p53 can induce programmed cell death through transactivation-independent mechanisms. We were intrigued by these studies, as their further investigation could potentially lead to novel and tractable ways of inducing selective cell death for therapeutic gain. One report in particular showed that a construct expressing only the first 214 aa from mouse p53 (termed d1214) could induce apoptosis without the activation of p53 target genes (15). We decided to investigate whether a similar truncation of human p53 would function in an analogous manner (Figure 1A). We began by expressing the first 210 aa from WT human p53 in p53-null Saos2 cells in order to rule out effects of endogenous p53, but this did not induce programmed cell death despite successful transfection (data not shown). Subsequent Western blot analysis demonstrated, however, that this truncation was not expressed at detectable levels (data not shown). In order to stabilize this truncation and 

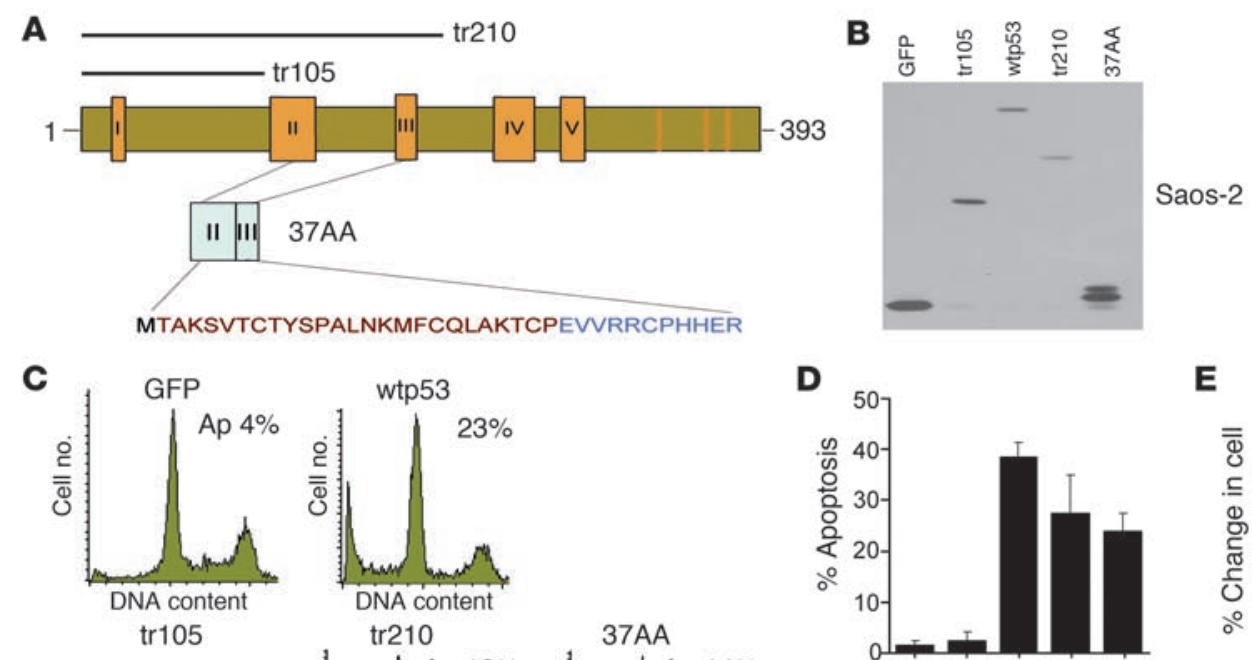

\section{D}

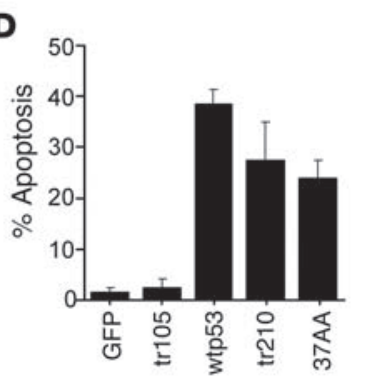

$\mathbf{E}$
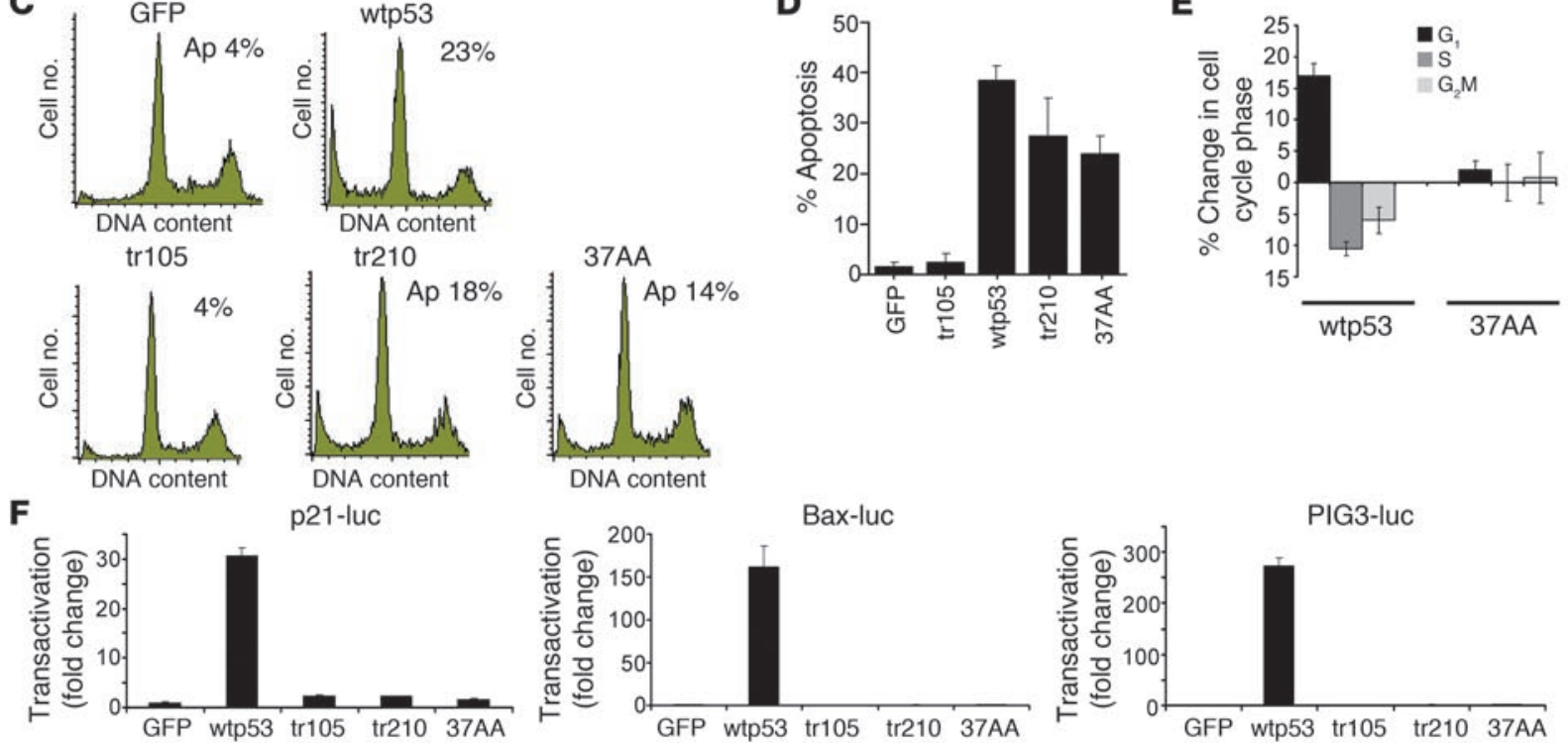

Figure 1

The p53-derived peptide 37AA induces cell death without direct activation of p53 target genes. (A) Representation of the p53 mutants used in this study. Full-length p53 is shown in green with 5 evolutionarily conserved regions (boxes) shown in yellow. Two truncations, tr105 (aa 1-105) and tr210 (aa 1-210), are shown as well as the composition of the 37AA peptide, with aa from conserved box II (residues 118-142) in red and those from conserved box III (residues 171-181) in blue. (B-E) Cells were transiently transfected with the indicated plasmids and subsequently analyzed 36 hours (C and E) and 48 hours later (D) for changes in cell cycle and cell death by flow cytometry. Ap, apoptosis. (B) Cells were transiently transfected with the indicated plasmids, and transgene expression was determined by Western blotting. The additional lower band seen following expression of 37AA represents expression from an alternate internal methionine. Mutation of this methionine to alanine resulted in expression of the larger protein species only and produced identical results (not shown). (F) No direct activation of p53 target genes by $37 \mathrm{AA}$ was observed. Saos-2 cells were transfected with luciferase reporter plasmids for the p53 target genes p21, Bax, and PIG3 as well as with the indicated expression constructs. After 24 hours, cells were assayed for luciferase activity, and the data were normalized against transfected $\beta$-gal activity. Values represent fold activation relative to activity of GFP alone, which was assigned as 1.

for ease of detection, we fused the 210 -aa domain to GFP. This created a fusion protein (referred to here as tr210) that not only was effectively expressed when transiently transfected into Saos- 2 cells (Figure 1B), but also resulted in accumulation of cells with sub- $\mathrm{G}_{1}$ content (Figure 1C), a verified measure of apoptotic rate (16). Cell death also occurred when a construct expressing WT p53 fused to GFP (referred to here as wtp53) was transfected into Saos-2 cells. Conversely, no death was observed following expression of a GFP fusion protein containing the first 105 aa from p53 (referred to here as tr105), even though this protein was expressed at a level higher than that of $\operatorname{tr} 210$ (Figure 1, B and C), which indicates that the death seen from tr210 was not caused solely by overexpression of a GFP fusion protein. Concordantly, removal of GFP from tr210 and $\operatorname{tr} 105$ and subsequent fusion of the truncations to a portion of the luciferase gene gave similar results, indicating that cell death was dependent on the first 210 aa of p53 and not on the generation of the GFP fusion in tr210 (data not shown).
Since fusion to GFP resulted in stable proteins, we were able to refine the apoptotic region of $\operatorname{tr} 210$. We found that in order to retain its full apoptotic potential, tr210 could only be refined $\mathrm{N}$-terminally to the beginning of the second evolutionarily conserved box of p53 (aa 118; data not shown). From the C terminus, refinement could only extend to the end of the third evolutionarily conserved box (aa 171; data not shown). Because these 2 evolutionarily conserved boxes are juxtaposed in the crystal structure of the p53 core domain (17), we tested whether the intervening sequence between the boxes could be removed without affecting the ability of the peptide to induce cell death. Indeed, when expressed in Saos-2, this peptide - composed of only 37 aa and referred to herein as 37AA (Figure 1A) - resulted in induction of cell death comparable to that observed for tr210 (Figure 1C). No cell death was seen, however, when either conserved box II or conserved box III was expressed independently (data not shown). The amount of cell death from 37AA increased with time (Figure 1D); however, 

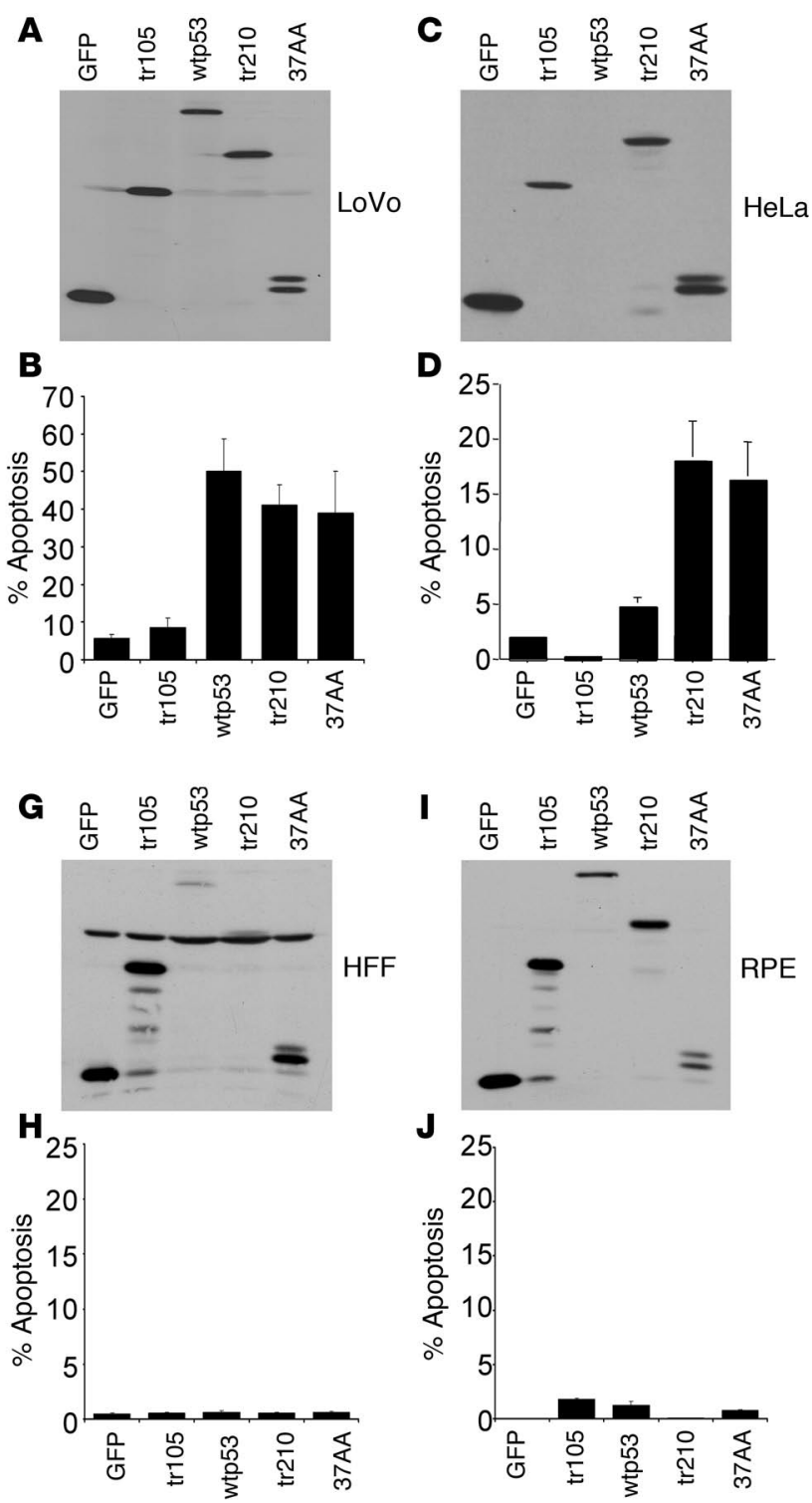

similar to the original mouse dl214 truncation (15), but different from wtp53, this increase in cell death was not accompanied by changes in cell-cycle status (Figure 1E).

We next sought to determine whether 37AA is defective in transactivation, as was found with the mouse dl214 truncation (15). We performed luciferase reporter assays, which measure direct transactivation potential, with constructs containing p53-responsive promoter fragments from the $p 21$ gene and from proapoptotic p53 target genes Bax and PIG3. In line with previous reports using these reporter constructs (18-20), wtp53 effectively transactivated the reporter in each case. However, 37AA was essentially inert in this assay, showing luciferase activity similar to that of cells transfected with GFP alone (Figure $1 \mathrm{~F}$ ). This result was identical to that previously reported with the mouse truncation dl214 (15) and indicates that 37AA induces cell death despite lacking the ability to directly transactivate $\mathrm{p} 53$ target genes.

We next tested whether the death-inducing activity of 37AA was specific to Saos-2 cells, or whether 37AA could kill other tumor cell lines. We expressed 37AA along with wtp53, tr210, and tr105

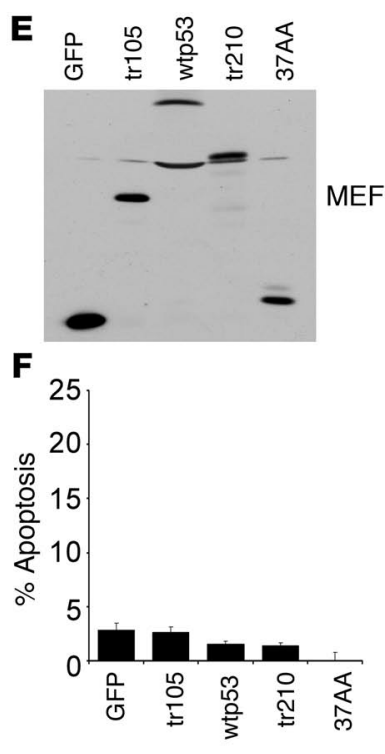

Figure 2

37AA causes selective killing of tumor cells. Tumor cell lines LoVo (A and B) and HeLa (C and $\mathbf{D})$ as well as primary cell cultures MEF (E and $\mathbf{F}), \operatorname{HFF}(\mathbf{G}$ and $\mathbf{H})$, and RPE (I and $\mathbf{J}$ ) were transfected or infected with the indicated transgenes. After 48 hours (transfection, $\mathbf{A}-\mathbf{D}$ ) or 72 hours (infection, $\mathbf{E}-\mathbf{K}$ ), transgene expression was assessed by Western blotting (A, C, E, G, and I), and the effects on cell death were determined by flow cytometry (B, D, F, H, J, and K).

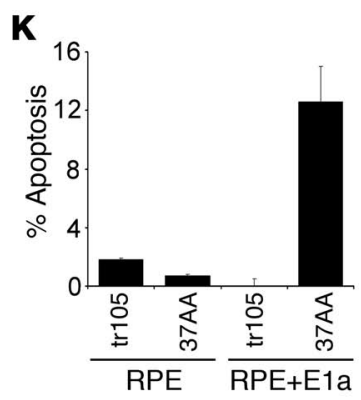

in 2 cell types that differ from Saos- 2 in different ways: LoVo cells, which are derived from a colonic adenocarcinoma and contain WT p53; and HeLa cells, which are cervical in origin and also contain WT p53. The p53 in HeLa, however, is rendered essentially null due to the presence of high-risk human papilloma virus (HPV), with the E6 protein of this virus causing p53 degradation (21). In both LoVo and HeLa cells, similar to our results in Saos-2 cells, 37AA caused apoptosis, as determined by the appearance of cells with sub- $\mathrm{G}_{1}$ DNA content, whereas minimal death was seen with tr105 (Figure 2, A and B). Interestingly, in the cases of Saos-2 and LoVo (Figure 1, C and D, and Figure 2B), the amount of death seen with 37AA was less than that seen with wtp53. In contrast, 37AA caused more death in HeLa cells than did wtp53 (Figure 2D). This presumably reflects the fact that wtp53 was degraded by the HPV-E6 protein, but 37AA was not (data not shown). Concordantly, although wtp53 expression was detected on longer exposures of the immunoblot (data not shown), the difference in expression of wtp53 and 37AA in HeLa cells was much greater than in the other cell lines analyzed (Figure 1B and Figure 2, C 
A
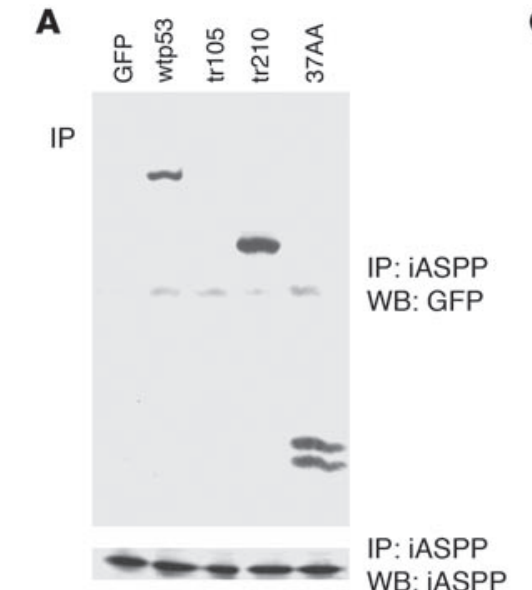

Input

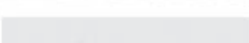

WB: IASPP

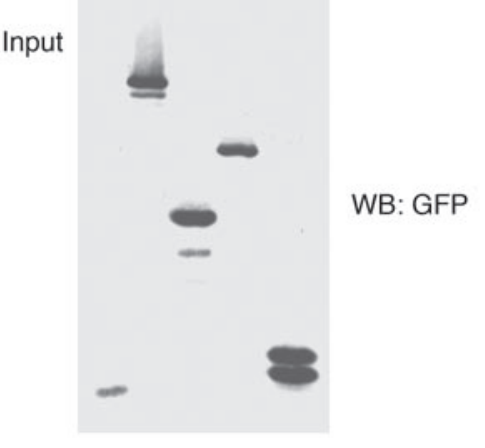

B

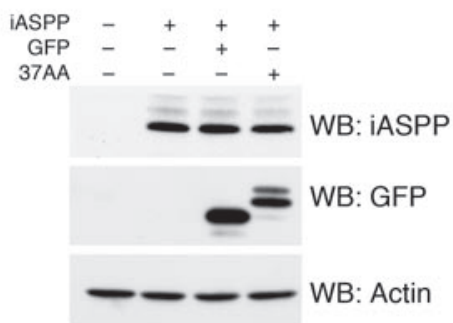

C
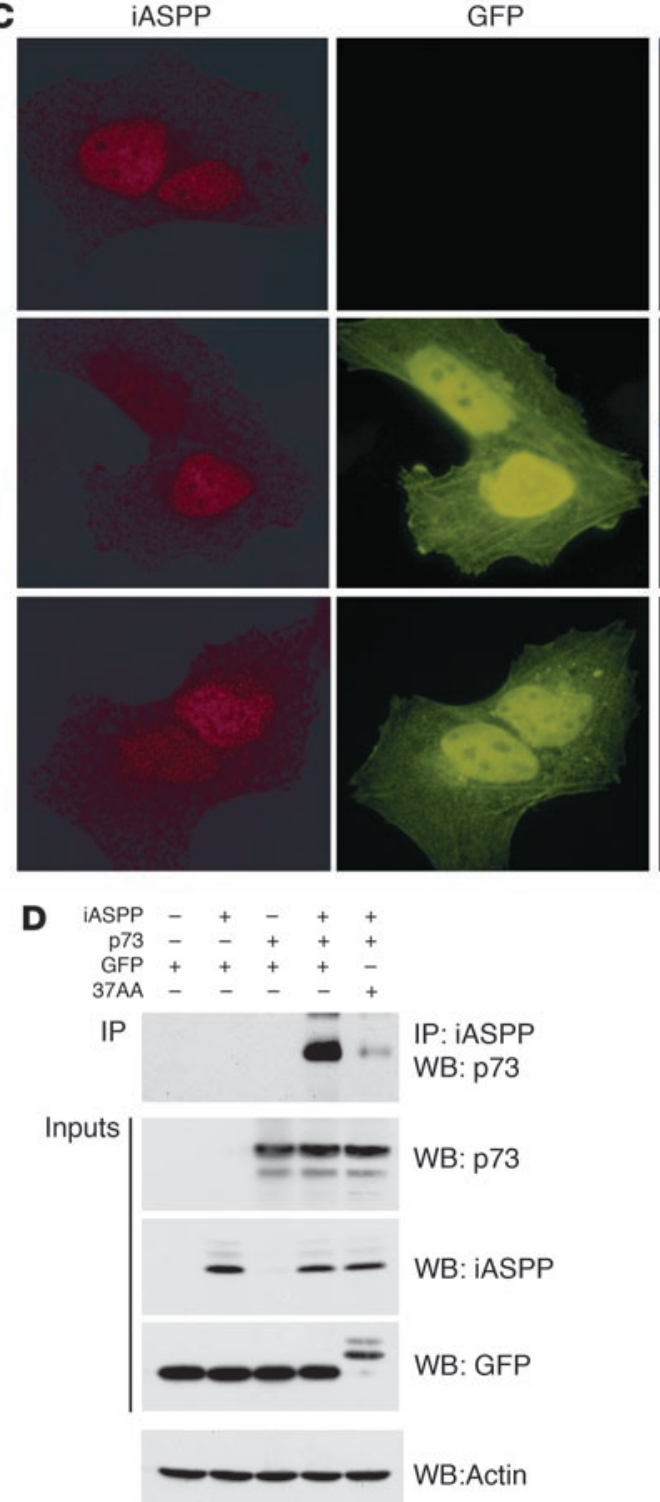
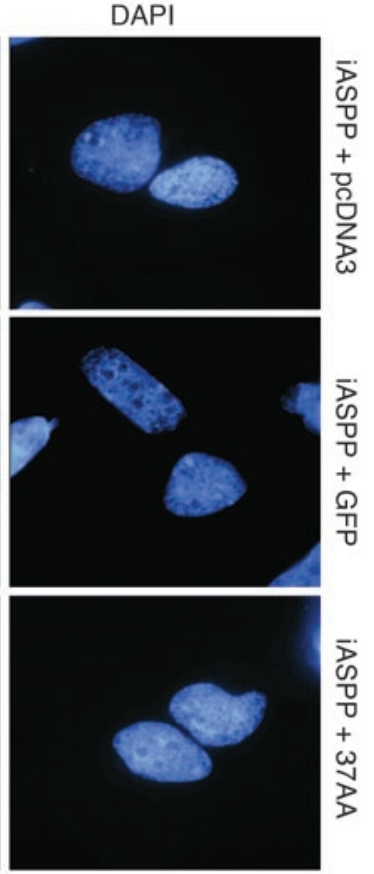

\section{(n)

(n)




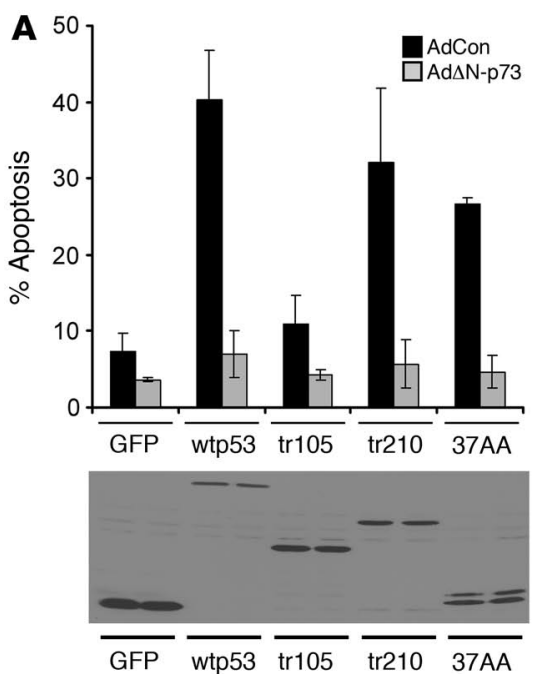

B
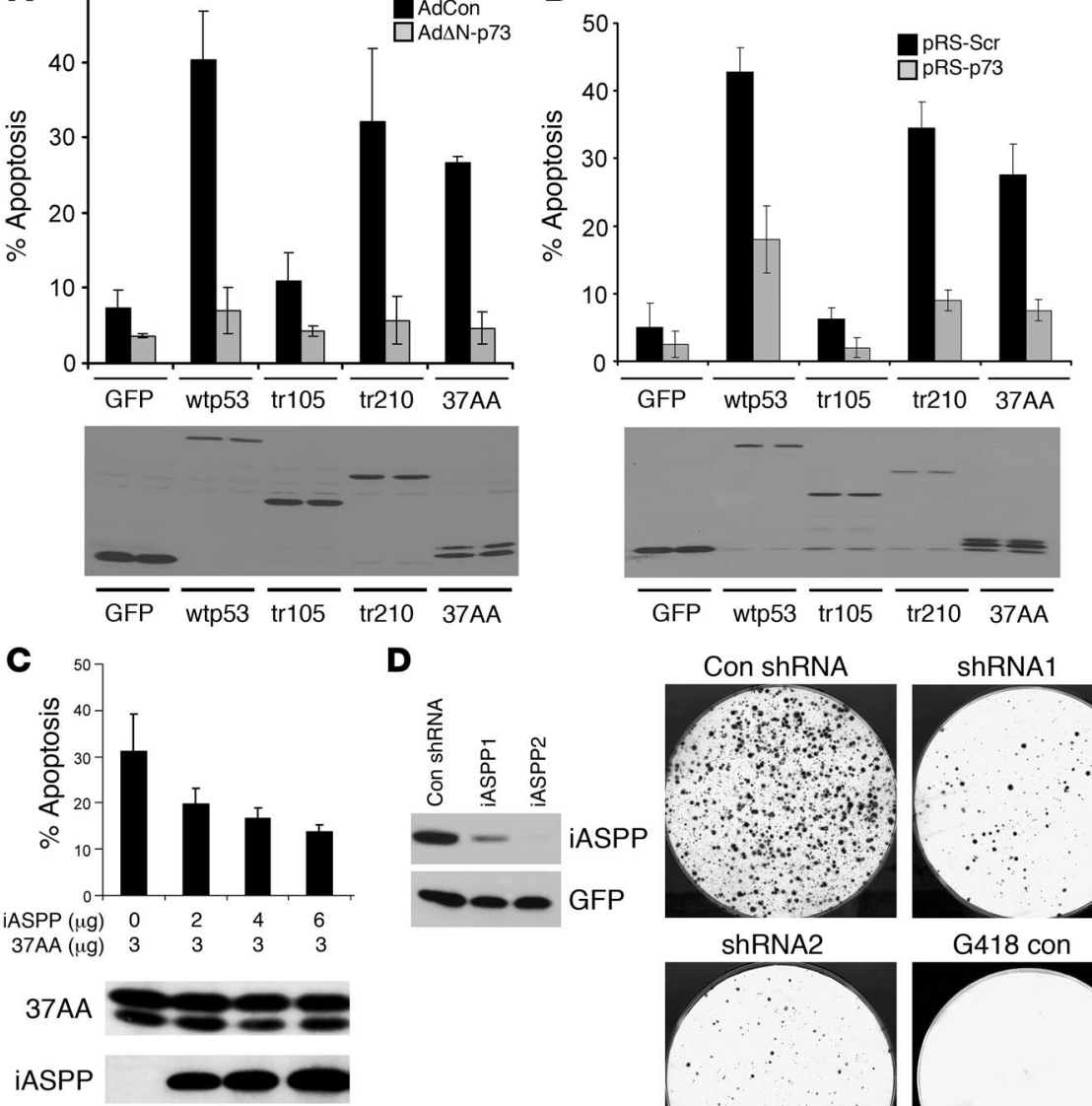

D

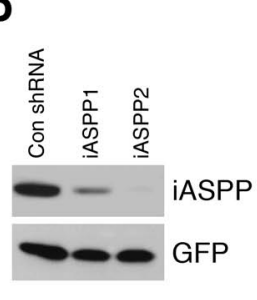

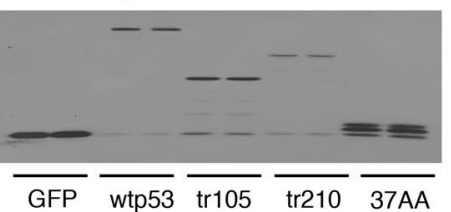

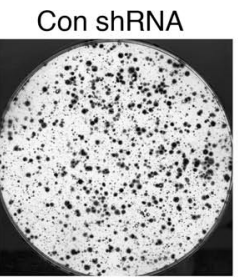
ShRNA2

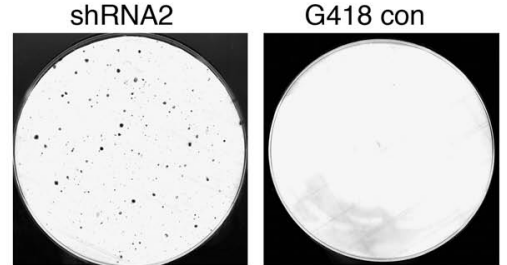

\section{Figure 4}

Expression of iASPP or interference with p73 inhibits 37AA-mediated cell death. (A) Saos-2 cells were transfected with the indicated plasmids and then infected with an adenovirus (Ad) expressing $\Delta \mathrm{N}-\mathrm{p} 73 \alpha$ or empty virus control (Con). After 48 hours, cells were analyzed for cell death by flow cytometry or for expression of transfected plasmids by Western blotting. (B) Saos-2 cells that had been infected with a retrovirus expressing either an shRNA directed against TA-p73 (pRS-p73) or a scrambled control (pRS-Scr) were transfected with the indicated plasmids. After 48 hours, cells were analyzed for cell death by flow cytometry or for expression of transfected plasmids by Western blotting. (C) Saos-2 cells were transiently cotransfected with the indicated amounts of 37AA and iASPP. After 48 hours, cells were analyzed for cell death by flow cytometry and for expression of transfected plasmids by Western blotting. (D) The effects of iASPP knockdown by RNA interference in p53-null cells were determined. Saos-2 cells were transfected with plasmids expressing 2 different shRNAs that target iASPP or a scrambled shRNA control. The effects of the shRNAs on transfected iASPP expression were determined by Western blotting after 24 hours, and the effects on long-term survival were determined by assessing the clonogenic capacity of the cells. G418 con, control for drug activity; cells were mocktransfected and selected with G418. Original magnification, $\times 1$. become markedly more sensitive to cell death induction by 37AA, indicating a selectivity in 37AA for killing oncogene-transformed cells (Figure 2K). In comparison, no sensitization to cell death was seen with tr105 (Figure 2K), despite its clearly detectable expression (data not shown).

tr210 and 37AA kill cells via inhibitory member of the ASPP family binding and p73. Because 37AA is composed of only 37 aa, it seems most conceivable that this molecule induces death through a proteinprotein interaction. Before searching for a binding protein through which 37AA mediates its effects, we first considered the plethora of proteins that have already been shown to bind p53. Above all, it was believed that not only must the binding protein bind in the region from which $37 \mathrm{AA}$ is derived, but since p53 can induce apoptosis in a wide range of tissues, the binding protein should also be ubiquitously expressed. Moreover, since our analysis of subcellular localization indicated that 37AA most likely functions in the nucleus (data not shown) and not in the cytoplasm or mitochondria, as has been proposed for other transactivation-independent cell mechanisms of p53, we considered that the binding protein through which 37AA induces death may also be a nuclear protein. One protein that fulfils these criteria is the apoptosis stimulating protein of p53 (ASPP) family member inhibitory member of the ASPP family (iASPP) (23). ASPP proteins have previously been shown to act as both positive (i.e., ASPP1 and ASPP2) and negative (i.e., iASPP) regulators of p53-induced apoptotic death (24,
25). Although ASPP1 and ASPP2 are predominantly cytoplasmic $(26,27)$, iASPP exists in both the cytoplasm and the nucleus (23), and we therefore hypothesized that if 37AA could bind this antiapoptotic member of the family, it may act to sequester iASPP and thereby permit death. We generated a FLAG-tagged form of iASPP and expressed this in cells together with 37AA and the other GFP fusion constructs previously described. Cell lysates were then subjected to IP with anti-FLAG antibody and analyzed by Western blotting for GFP. Not only was 37AA immunoprecipitated with iASPP, but so were wtp53 and tr210 (Figure 3A). No binding was seen, however, with the tr105 truncation, indicating that only the fusion proteins that induce cell death were immunoprecipitated in this assay for iASPP binding.

Although 37AA clearly bound iASPP, a previously described negative regulator of $\mathrm{p} 53$, we found in the present study that 37AA induced cell death in cells null for p53 (Figure 1B). However, ASPP proteins have also been shown previously to regulate cell death downstream of p53 family members p63 and p73 (24). We therefore considered whether 37AA, by binding iASPP, may result in derepression of p53 family members and thereby cause death. We began by testing whether 37AA could affect iASPP protein levels or iASPP localization such that downregulation of iASPP or relocalization of iASPP would diminish its antiapoptotic effects. While 37AA had no effect on the stability of iASPP (Figure 3B), it seemed to cause a partial relocalization of iASPP from the nucleus 

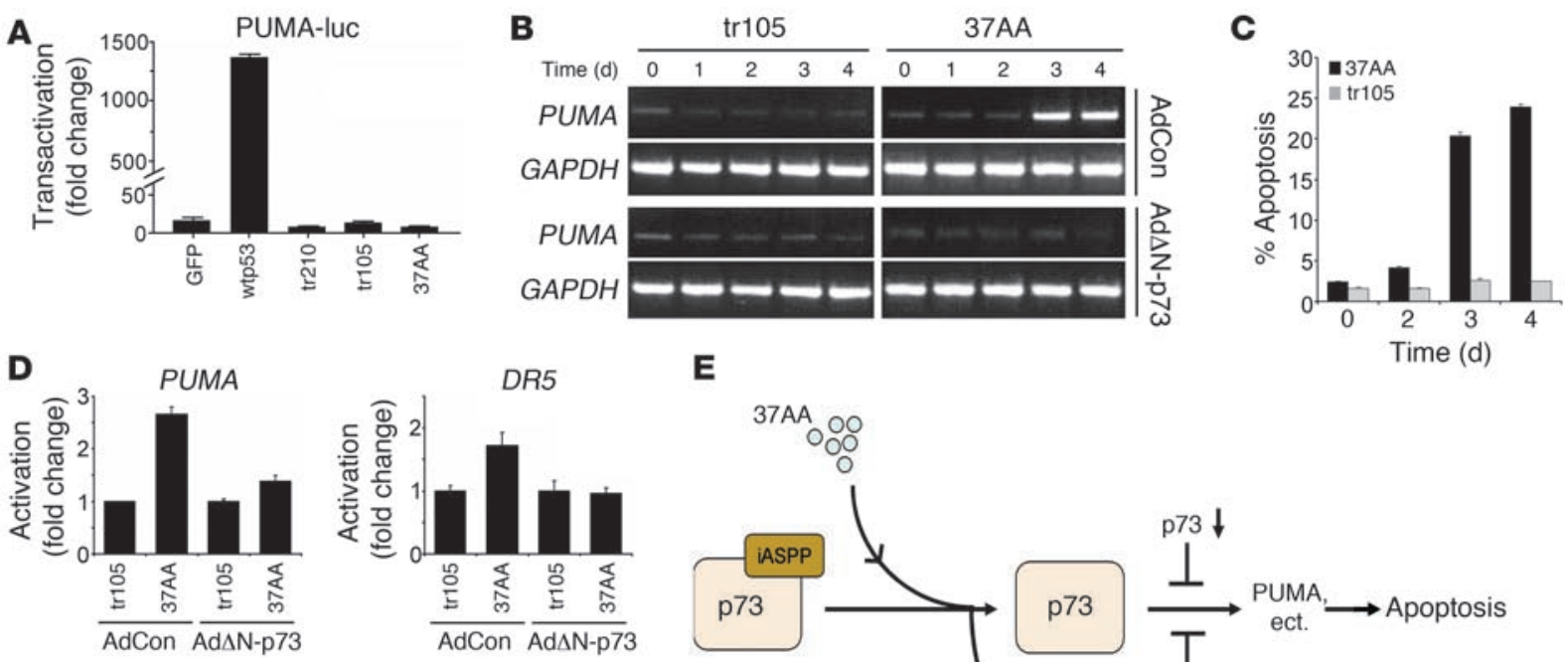

$\mathbf{E}$


\section{Figure 5}

37AA does not transactivate directly, but causes secondary activation through p73. (A) Saos-2 cells were transiently transfected with $5 \mu$ P PMA luciferase plasmid and $200 \mathrm{ng}$ each of the indicated expression constructs. After 72 hours, cells were assayed for luciferase activity, and the data were normalized against transfected $\beta$-gal activity. Values represent fold activation relative to the activity of GFP alone, which was assigned as 1 . (B) Saos-2 cells were infected as indicated with adenoviruses expressing tr105, 37AA, $\triangle N-p 73$ adenovirus, or control empty adenovirus. At the indicated time points, RNA was isolated from the cells and subjected to RT-PCR for PUMA and GAPDH. (C) At the same time cells were harvested and assessed for changes in cell death by flow cytometry. (D) Cells were infected with the indicated adenoviruses for 48 hours. The mRNA levels for PUMA, DR5, Bax, and p21 were determined by qPCR. Samples were normalized to the levels of $18 S$ ribosomal RNA. (E) Schematic of the mode of action of 37AA. Introduction of 37AA into p53-null cells sequesters iASPP and thereby derepresses p73. TA-p73 subsequently activates apoptotic target genes such as PUMA to bring about programmed cell death. In line with this model, cell death from $37 \mathrm{AA}$ can be inhibited by decreasing p73 activity or increasing iASPP.

to the cytoplasm (Figure 3C). As a result, we reasoned that by binding iASPP, 37AA may be disrupting the interaction of iASPP with p53 family members. Coimmunoprecipitation experiments were undertaken to test this using 37AA, iASPP, and p73. In the presence of GFP, p73 was clearly detected following IP of iASPP (Figure $3 \mathrm{D})$. However, this was greatly reduced in cells cotransfected with 37AA (Figure 3D). As no changes in the input levels of iASPP or p73 were observed, these data indicate that the iASPP-p73 complex can be disrupted by 37AA and raises the possibility that 37AA induces death through derepression of p53 family members.

To test for the involvement of p53 family members in 37AAinduced death, we used a naturally occurring form of $\mathrm{p} 73 \alpha$ lacking a transactivation domain, $\Delta \mathrm{N}-\mathrm{p} 73 \alpha$. Previous studies have shown that $\Delta \mathrm{N}-\mathrm{p} 73 \alpha$ has the capacity to inhibit the activity of various transcript variants of TA-p73 as well as transactivation-competent forms of $\mathrm{p} 63$ (TA-p 63) and $\mathrm{p} 53$, and is therefore a broad-spectrum inhibitor of the p53 family (28). Indeed, expression of $\Delta \mathrm{N}-\mathrm{p} 73 \alpha$ reduced the amount of cell death observed following expression of 37AA (Figure 4A). In the absence of $\Delta \mathrm{N}-\mathrm{p} 73 \alpha, 37 \mathrm{AA}$ induced apoptosis at a rate of about $25 \%-30 \%$ when expressed for 48 hours. When $37 \mathrm{AA}$ was coexpressed with $\Delta \mathrm{N}-\mathrm{p} 73 \alpha$, however, apoptosis was reduced to basically background levels (less than 5\%). As would be expected, in line with a previous study suggesting essential roles for p63 and p73 in p53-induced death (29), a similar effect of $\Delta \mathrm{N}$-p $73 \alpha$ was also seen following coexpression with $\mathrm{p} 53$, with apoptosis again being reduced to background levels (Figure 4A). Because the effects on cell death are pleiotropic in these experiments, this does raise the question of whether $\Delta \mathrm{N}-\mathrm{p} 73 \alpha$ inhibits $37 \mathrm{AA}$-induced death in a specific manner, or whether $\Delta \mathrm{N}-\mathrm{p} 73 \alpha$ is simply a broad suppressor of programmed cell death. The fact that no effect of $\Delta \mathrm{N}$-p $73 \alpha$ expression was seen during Bax-induced death, however, indicates that this suggests the latter is not the case (data not shown).

As $\Delta N-p 73 \alpha$ can inhibit the effects of both TA-p63 and TA-p73, our results with this construct do not discern whether 37AAinduced cell death is mediated by $\mathrm{p} 63$, by $\mathrm{p} 73$, or by both equally. Because 73 has a more documented role in cell death and chemosensitivity (13), we decided to analyze more directly the role of p73 in 37AA-induced death. We used a previously described RNA interference construct designed to specifically knock down TA variants of p73 (30). Saos-2 cells were retrovirally infected with this construct (pRS-p73) or with a construct expressing a scrambled short hairpin RNA (shRNA construct; pRS-Scr). Subsequent transfection of these cells with 37AA revealed that while cells infected 

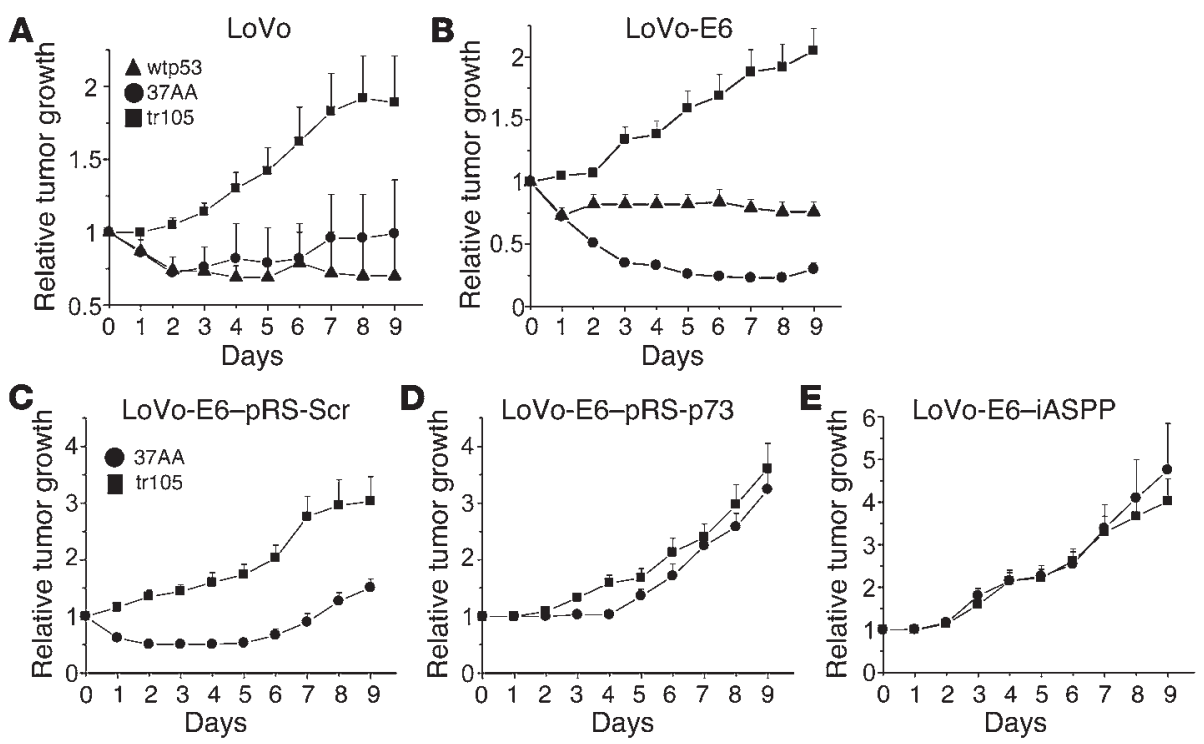

\section{Figure 6}

$37 \mathrm{AA}$ causes tumor regression in vivo. Cultures of LoVo (A), LoVo-E6 (B), LoVoE6-pRS-Scr (C), LoVo-E6-pRS-p73 (D), and LoVo-E6-iASPP cells (E) were injected into the flanks of athymic mice. Upon tumor formation (day 0), mice were treated by intravenous tail vein injection on days $0,2,4,6$, and 8 with DNA-dendrimer complexes containing $50 \mu \mathrm{g}$ of 37AA, wtp53, or tr105. Tumor volume was measured daily, and growth was plotted as relative to tumor size on day 0 . with the scrambled shRNA died in a manner similar to that of parental Saos- 2 cells (see Figure 1B), those infected with pRS-p73 were completely resistant to death from 37AA, with levels of apoptosis comparable to those of tr105 or GFP alone (Figure 4B). These data essentially parallel the results obtained with $\Delta \mathrm{N}-\mathrm{p} 73 \alpha$ and therefore clearly show that in these cells, 37AA induces death by a mechanism dependent on TA-p73.

If our hypothesis was correct that 37AA was serving to sequester iASPP away from $\mathrm{p} 73$, cell death should also be inhibited by increasing the levels of iASPP. To test this, constant levels of 37AA were expressed in Saos- 2 cells together with increasing amounts of iASPP. Following transfection, cells were harvested and analyzed for cell death by flow cytometry. Excess exogenous iASPP indeed inhibited cell death induced by 37AA (Figure 4C). When expressed alone, 37AA ( $3 \mu \mathrm{g})$ induced approximately $30 \%$ apoptosis following expression for 48 hours. This was reduced to $12 \%$ when 37AA was coexpressed with $6 \mu \mathrm{g}$ iASPP. Conversely, if the cell death seen was solely due to iASPP sequestration and there were no other signals from 37AA, cell death should also be evident as a result of iASPP depletion. We therefore generated shRNA constructs to knock down iASPP. These shRNA constructs caused a marked reduction in the amount of iASPP protein expressed from an iASPP expression vector when compared with the effects of a scrambled shRNA control (Figure 4D). Transient transfection of these constructs into Saos- 2 cells, however, did not result in detectable cell death in the short term, as assessed by flow cytometry - presumably due to the long half-life of the endogenous pool of iASPP protein (data not shown). However, when expressed continually over a period of 2 weeks to allow for depletion of endogenous iASPP, these constructs caused a considerable decrease in the clonogenic potential of the cells compared with cells transfected with scrambled shRNA control (Figure 4D). Consistent with our observations using $37 \mathrm{AA}$, these results confirm that perturbing the function - or in this case, the levels - of iASPP can result in a decrease in the viability of p53-null cells.

$37 A A$ causes transactivation via $p 73$. Although our previous analysis of the transactivation capacity of 37AA indicated it could not activate p53 target genes directly (Figure 2), we considered that the removal of iASPP from $\mathrm{p} 73$ may result in secondary gene acti- vation via $\mathrm{p} 73$. This secondary activation may not be evident in luciferase reporter assays, as they generally measure the activity of an excess of transfected expression plasmid on an excess of transfected reporter plasmid. The possibility of transactivation occurring via a relatively small amount of a cellular intermediary (p73) is therefore unlikely. We reasoned that if secondary activation was occurring during 37AA-induced death, we may be able to detect it by examining endogenous target gene activation - an excess of transfected 37AA could activate a relatively small amount of cellular p73, which could then have the capacity to activate the cellular promoter. To test this possibility, we analyzed activation of p53 upregulated modulator of apoptosis (PUMA), a target gene of both p53 and p73 (31-33), in p53-null H1299 cells, which are known to induce p73 in response to treatment with cisplatin (34). While treatment with cisplatin caused a p73-dependent induction of endogenous PUMA, this was not reflected in activation of a transfected PUMA luciferase construct, which was present in comparatively vast excess in the cells (Supplemental Figures 1 and 2; supplemental material available online with this article; doi:10.1172/JCI29820DS1). Further analysis of this PUMA reporter indicated, as was seen with our analysis of other target genes (Figure 2), that the reporter was strongly activated by wtp53 but not by 37AA (Figure 5A). However, infection of Saos- 2 cells with an adenovirus expressing 37AA resulted in strong activation of endogenous PUMA, whereas no activation was seen with tr105 (Figure 5B). This induction of PUMA occurred concomitantly with the ability of 37AA to induce cell death (Figure 5C), and, as with 37AA-induced death, dominant-negative $\triangle \mathrm{N}$-p73 abolished the induction of PUMA, indicating that this indeed represents a secondary activation via p73 (Figure 5B). As a result, we tested whether other previously described p 73 target genes are activated by $37 \mathrm{AA}$ and also whether this activation could be deleted after adenoviral administration at a time point that just precedes death (48 hours). Since the level of activation of target genes at this time point may be below the threshold required for detection by semiquantitative RT-PCR, mRNA levels were analyzed by quantitative real-time PCR (qPCR). Not only was activation of PUMA evident at this time point, but mRNA for death receptor 5 (DR5; ref. 35) was also induced (Figure 5D). The induction levels 
were similar to those following infection of cells with TA-p73 or following treatment with cisplatin - an agent known to activate endogenous p73 (Supplemental Figure 2; ref. 34). Moreover, consistent with a role for $\mathrm{p} 73$ in $37 \mathrm{AA}$ action, activation of both of these targets was greatly impeded when cells were infected with dominant-negative $\Delta \mathrm{N}-\mathrm{p} 73$ (Figure 5D). However, we saw no activation of $B a x$ (another proapoptotic target of p53 and p73; Figure $5 \mathrm{D})$, indicating that 37AA-induced, $\mathrm{p} 73$-mediated gene activation may be selective for certain target genes. However, the extent to which WT p53 and TA-p73 can activate Bax needs to be determined before any conclusions can be drawn about the regulation of Bax in these cells.

In addition to analysis of the activation of apoptotic target genes, we also analyzed the activation of the cell-cycle regulator $p 21$. As ASPP proteins are considered to selectively regulate activation of apoptotic genes, we were surprised to observe that 37AA caused activation of $p 21$ (Figure 5D). This activation was not dependent on $\mathrm{p} 73$, however, and the nature of this induction - whether mechanistically linked to 37AA, possibly through a p73-independent mechanism, or simply a response to 37AA action - therefore remains unclear. The induction of $p 21$ by $37 \mathrm{AA}$ also seems to be inconsistent with the fact that 37AA did not affect cell-cycle progression (Figure 1E). Analysis of p21 protein levels revealed that this change in p21 mRNA was not reflected in changes in p21 protein (data not shown), proving an explanation for this apparent paradox. Taken together, therefore, our data suggest a model wherein p53-null cells, 37AA binds to iASPP, resulting in the derepression of $\mathrm{p} 73$. Therefore, even in the absence of other signals, p73 is free to activate apoptotic target genes such as PUMA and $D R 5$ to invoke programmed cell death (Figure 5E).

$37 A A$ causes tumor regression in vivo. Since 37AA can cause cell death in multiple tumor cells types in vitro, we next questioned whether 37AA would work as a therapeutic agent in vivo to cause selective cell death and tumor regression. As Saos- 2 cells are nontumorigenic in athymic mice, we decided to analyze the effects of 37AA on tumors formed with LoVo cells, which are frequently used in xenograft studies. Cultures of LoVo cells were therefore injected into the flanks of mice to induce tumor formation. Once tumors were palpable, mice were treated by intravenous injection of 37AA, wtp53, or tr105 expression plasmids using a nanoparticle nonviral delivery system involving complexes of DNA with polypropylenimine dendrimers. This system has been developed by us and by others and has been shown to deliver highly tumor-specific expression of a transgene and, where a death-inducing gene is used, tumor regression with limited toxicity $(36,37)$. Moreover, our present studies showed that tail vein injection of DNA-dendrimer complexes containing CMV-driven $\beta$-gal can induce transgene expression within the tumor comparable to that seen following intratumoral injection (Supplemental Figure 3). When coupled with the ability of this systemically delivered agent to potentially treat disseminated disease, this makes this approach an exciting prospect for nonviral anticancer gene medicine.

Animals bearing tumors were therefore treated on days $0,2,4$, 6 , and 8 relative to the day of tumor palpability with dendrimers containing $50 \mu \mathrm{g}$ of wtp53, tr105 or 37AA. Analysis of tumor size during these treatments revealed that while tumors treated with tr105 exhibited transgene delivery and yet continued to grow (Figure 6A and Supplemental Figure 4), tumor growth in mice treated with wtp53 and 37AA was greatly impeded, with marked tumor regression occurring in both cases (Figure 6A). The effects of DNA-dendrimer complexes on animal body weight were minimal and similar following all treatments (Supplemental Figure 5). Since LoVo cells contain endogenous WT p53, we next sought to determine whether the effects of 37AA on tumor regression were dependent on p53. To inactivate p53, therefore, LoVo cells were infected with a retrovirus expressing HPV-E6, which causes the degradation of p53 (21) but not p73 (38). These cells were then injected into mice and treated with DNA-dendrimer complexes as before. As shown in Figure 6B, 37AA was still very effective against tumors formed from LoVo-E6. In contrast, due to the presence of E6 (and consistent with what we observed in vitro in HeLa cells; Figure 1D), the effectiveness of dendrimers containing wtp53 was greatly compromised compared with that in WT LoVo cells (Figure 6, A and B). This demonstrates not only that 37AA is able to cause tumor regression in the presence and absence of endogenous p53, but also that, in terms of gene therapy, it seems to be effective in situations where the therapeutic potential of delivering WT p53 would be greatly compromised.

We next considered whether, in the absence of p53, 37AA was causing therapeutic effects in vivo that could be affected by modulating the levels of p73 and iASPP. To do this, we generated a LoVoE6 cell line that expresses the TA-p73-targeting shRNA construct we had used in our in vitro studies. When compared to tumors generated with LoVo-E6 cells containing a scrambled shRNA as control (LoVo-E6-pRS-Scr), those generated with the shRNA to target TA-p73 (LoVo-E6-pRS-p73) were markedly resistant to the therapeutic effects of 37AA (Figure 6, C and D). This loss of therapeutic response was also evident in cells that had been engineered to overexpress iASPP (LoVo-E6-iASPP), with tumor growth in 37AA-treated mice essentially identical to that of mice treated with tr105 (Figure 6E). Taken together, these results are consistent with the view that the therapeutic effects of 37AA occur by a mechanism in line with the model we established based on our in vitro results (Figure 5E).

\section{Discussion}

To our knowledge, this study represents the first report of a mechanism to directly target $\mathrm{p} 53$ family members for therapeutic gain. We have demonstrated that expression of the 37AA hybrid peptide from p53 was capable of inducing apoptosis of cultured cells in vitro regardless of p53 status and, concordantly, that nanoparticle delivery of this peptide causes tumor regression in vivo by a mechanism dependent on $\mathrm{p} 73$. Human tumors exhibit a very low frequency of 773 mutation, and consequently 37AA or its functional mimetics may have a broad therapeutic role for the treatment of malignant disease, although it must be considered that not all tumors will express p73. Functional molecular analysis revealed that $37 \mathrm{AA}$ acts via sequestration of $\mathrm{AASPP}$, and we have previously shown that iASPP functions as a negative regulator of p53, p73, and possibly p63 (39). Sequestration or inhibition of iASPP may therefore have even broader therapeutic value. For example, in situations where p73 is absent, it would be predicted that interference with iASPP binding or function may well induce cell death by $\mathrm{p} 53, \mathrm{p} 63$, or both, if they are present. Moreover, when all p53 family members are present, one might predict an even stronger cell death response.

A number of recent reports have indicated that iASPP is overexpressed in breast cancers and certain leukemias $(39,40)$. It seems possible that in these cases, iASPP overexpression may be involved in both the establishment and the maintenance of tumors. 
In terms of therapy, this could therefore be extremely important if, as we predict, iASPP serves to suppress signals that promote cell death. In this regard, although p73 was not analyzed in these studies, it is noteworthy that increased iASPP expression in breast cancer exhibited significant reciprocity with mutation of p53 (39). Therefore, in these tumors, iASPP may be acting in an enhanced manner to inhibit p53-mediated cell death responses, thereby circumventing the pressure to mutate $\mathrm{p} 53$ directly. In the leukemia study, there was no evident relationship to p53 status, indicating that iASPP overexpression may promote leukemogenesis by a mechanism that does not involve p53 inactivation, and it is tempting to speculate that this may involve inactivation of p73. Either way, we predict that if the establishment and maintenance of these tumors is intrinsically dependent on iASPP, this may widen the therapeutic window of iASPP inhibition and indicate that these tumors would be hypersensitive to the effects of 37AA or its mimetics.

Finally, it is worthy to consider how 37AA or other subsequently derived agents that perturb iASPP function may work in conjunction with chemotherapeutic agents that are currently in the clinic. It is clear from our present results that dendrimer-delivered 37AA cannot cause complete tumor regression and that tumors regrow with time (Figure 6). Although dendrimer administration is very tumor specific, only about $25 \%$ of the tumor cells expressed the transgene following each administration (Supplemental Figure 4). Therefore, there is clearly room for the improvement of dendrimer technology in general. In terms of this study specifically, it is particularly notable that a number of drugs have previously been shown to increase the levels of $\mathrm{p} 73$, and $\mathrm{p} 73$ has been shown to be important for chemosensitivity in response to these drugs $(13,41,42)$. It is not known, however, whether these agents disturb the interaction of P73 with iASPP, although our initial observations indicated that 37AA did not affect the levels of p73 (data not shown). It therefore remains possible that interference of iASPP in combination with these drugs may result in an enhanced therapeutic response.

As with any rationally designed, molecularly targeted therapy, profiling of tumors will always be a prerequisite to determining the likely effectiveness of this strategy, which we believe to be novel, in any given tumor. For example, tumors overexpressing $\Delta \mathrm{N}$-forms of $\mathrm{p} 73$ that lack a transactivation domain could potentially be resistant to iASPP perturbation (28). However, in light of the provocative observations presented here, there appears to be clear value in the further investigation of 37AA or its derivatives as agents for cancer therapy.

\section{Methods}

Plasmids. The plasmids EGFP-N1wtp53, EGFP-N1tr105, EGFP-N1tr210, and EGFP-N137AA were made by inserting wtp53, tr105 (residues 1-105 from wtp53), tr210 (residues 1-210 from wtp53), and 37AA (fusion of residues 117-142 and 171-181 from wtp53) fragments into pEGFP-N1 (Clontech) using EcoRI and BamHI restriction sites. pcDNA3V5iASPP has been described previously (43). Flag-iASPP (RAI splice variant) was cloned into $\mathrm{PCB}^{+}$from cDNA with primers containing an $\mathrm{N}$-terminal FLAG tag and KpnI and NotI restriction sites. An EcoRI/EcoRV fragment from this plasmid containing the FLAG-iASPP insert was then cloned into pLPC to generate pLPC-iASPP. pShuttleCMV-GFP, pShuttleCMVp53, pShuttleCMV-tr105, pShuttleCMV-tr210, and pShuttleCMV-37AA were made by inserting GFP, wtp53, tr105, tr210, and 37AA fragments into pShuttleCMV (Stratagene) using BgIII and NotI restriction sites. pShuttleCMV-HA-TA-p73 $\alpha$ and pShuttleCMV-HA- $\Delta$ N-p73 $\alpha$ were created by cloning HA-TA-p $73 \alpha$ and HA- $\Delta \mathrm{N}-\mathrm{p} 73 \alpha$ fragments from
pcDNA3-HA vectors (kindly donated by T. Crook, Breakthrough Breast Cancer, London, United Kingdom) into the pShuttleCMV vector using KpnI and XhoI restriction enzymes. EGFP-N1-PUMA has been described previously (31). The reporter constructs for PIG3 and p21 and PUMA were kindly provided by B. Vogelstein (Howard Hughes Medical Institute and Johns Hopkins University, Baltimore, Maryland, USA) and have been described previously $(18,19,32)$. The reporter construct for Bax was kindly provided by J. Reed (Burnham Institute for Medical Research, La Jolla, California, USA) and has also been described previously (20). pJ $3 \Omega \beta$-gal has been previously described (16). pWZLBlast-E6 was generated by PCR amplification of E6 sequences to include a 5' FLAG tag and BamHI restriction sites. Amplified sequences were cloned into the BamHI site of pWZLBlast.

Cell culture, transfection, and retroviral infection. Saos-2, Saos-2-pRS-Scr, Saos-2-pRS-p73, HeLa, H1299, MEF, HFF, RPE, RPE-E1a, LoVo, LoVo-E6, LOVO-E6-iASPP, LoVo-E6-pRS-Scr, and LoVo-E6-pRS-p73 cells were grown in DMEM supplemented with $10 \%$ fetal bovine serum and maintained at $37^{\circ} \mathrm{C}$ in an atmosphere of air and $5 \% \mathrm{CO}_{2}$. All cells except Saos-2-pRS-Scr, Saos-2-pRS-p73, RPE-E1a, LoVo-E6, LoVo-E6-iASPP, LoVo-E6-pRS-Scr, and LoVo-E6-pRS-p73 were obtained from ATCC. For tumor-tracking purposes, all LoVo lines expressed GFP. MEFs were isolated from day E13.5 C57BL/6 mouse embryos using standard techniques. Saos-2-pRS-Scr, Saos-2-pRS-p73, and LoVo-E6 cells were generated by infection with pRetroSUPER-Scr (pRS-Scr), pRetroSUPER-p73 (pRS-p73) (Oligoengine), and pWZLBlast-E6, respectively. Secondary infections of LoVo-E6 with pLPC-iASPP, pRS-Scr, and pRS-p73 were performed to generate the lines LOVO-E6-iASPP, LoVo-E6-pRS-Scr, and LoVo-E6-pRS-p73, respectively. Retroviruses were generated by transfection of Phoenix packaging cells (G. Nolan, Stanford University, Stanford, California, USA). Infective supernatants were then administered to target cells followed by appropriate drug selection as previously described (44). Calcium phosphate precipitation was used to transiently transfect cells as previously described (16). Cells were harvested at the times indicated in the legends to Figure 1, B-F, Figure 2, A-D, Figure 4, A and B, and Figure $5 \mathrm{~A}$. For cell death and luciferase assays, cells were respectively plated prior to transfection at $0.8 \times 10^{6}$ and $0.5 \times 10^{6}$ cells per 9 -cm dish. Assays were conducted as previously described (16).

Western blotting and IP. Cells were lysed in a RIPA buffer, and protein was quantified using a BCA assay (Sigma-Aldrich). For each IP, 1,000 $\mu \mathrm{g}$ protein lysate was used; for Western blot, $50 \mu \mathrm{g}$ protein was used per lane. The antibodies used were as follows: HA-tagged anti-rabbit polyclonal antibody (Upstate USA Inc.), FLAG-tagged anti-M2 monoclonal antibody (SigmaAldrich), anti-V5 (Invitrogen) and anti-GFP full-length Aequorea victoria peptide polyclonal antibody (BD Biosciences). For IP, iASPP was transiently transfected into Saos-2 cells. Where indicated in Figure 2, A, B, and D, cells were cotransfected with or infected respectively with plasmids and adenoviruses expressing p73 or p53-GFP fusions. For adenoviral infections, cells were washed 3 times in serum free medium and adenoviruses were added at a concentration of 12 infectious units per cell for 12 hours. FLAG-tagged antiM1 agarose affinity gel was used for precipitating FLAG-tagged protein.

Generation of adenoviruses. Linearized Shuttle plasmids were electroporated into BJ5183-AD-1 electrocompetent cells (Stratagene) containing the Adeasy- 1 adenoviral construct. Recombinant plasmids were then amplified in XL10-Gold cells (Stratagene) and transfected into HEK293 cells after restriction digest with PacI. Following infectious adenovirus amplification in HEK293 cells, purified virus was isolated by freeze-thaw extraction and titered using the BD Biosciences Adeno-X Rapid Titer kit. Adenoviruses were added to cultures at a concentration of 12 infectious units per cell.

RT-PCR and $q$ PCR. RT-PCRs for PUMA were performed using the QIAGEN Proofstart PCR kit with Q-solution and $1 \mu \mathrm{l}$ proofstart DNA polymerase per 
reaction. The primers used were 5 '-GCTGGCGGAGCAGCACCTGGAGT-3' (forward) and 5'-GGCTCTGTGGCCCCTGGGTAA-3' (reverse). These produce a fragment of $287 \mathrm{bp}$. The PCRs were run for 28 cycles at annealing temperature of $62^{\circ} \mathrm{C}$ and 1 -minute extension cycle. RT-PCRs for GAPDH were performed using the PCR core kit (Applied Biosystems). The primers used were 5' -ACCACAGTCCATGCCATCAC-3' (forward) and 5'-TCCACCACCCTGTTGCTGTA-3' (reverse). These produce a fragment of $580 \mathrm{bp}$. The PCRs were run for 21 cycles at annealing temperature of $66^{\circ} \mathrm{C}$ and 1 -minute extension cycle. qPCR analysis was carried using the DyNAmo SYBR Green 2-step qRT-PCR kit (Finnzymes). Validated QuantiTect primer sets for p21, PUMA, DR5, Bax, and 18S ribosomal RNA (QIAGEN) were used. Data collection was carried out using a Chromo4 real-time PCR detector and analyzed with Opticon Monitor 3. qPCR cycling parameters were 15 minutes at $95^{\circ} \mathrm{C} ; 34$ cycles of 10 seconds at $94^{\circ} \mathrm{C}, 30$ seconds at $55^{\circ} \mathrm{C}$, and 30 seconds at $72^{\circ} \mathrm{C}$; and 10 minutes at $72^{\circ} \mathrm{C}$. Expression levels of genes analyzed by qPCR were normalized to $18 \mathrm{~S}$ ribosomal RNA levels.

RNA interference and cell viability assays. iASPP shRNA target sequences were determined using the Oligoengine online design tool. One scram$\mathrm{bled} / \mathrm{random}$ sequence was also designed. The chosen sequences were iASPP shRNA1-ACAGTACCAGCAGATCATC, iASPP shRNA2-CTACTCTATCGTGGATTTC, and a random shRNA-ACTGATCTGCATGTATT$\mathrm{GG}^{-}$. DNA oligos were then designed with the appropriate hairpin and restriction sites added. The DNA oligos were annealed and cloned into the pRetroSUPER-neo-GFP vector following manufacturers instructions. Following sequencing, the effectiveness of the constructs to knockdown iASPP was determined by transient cotransfection of each shRNA with FLAG-iASPP. iASPP levels were then determined by Western blotting after 24 hours. To test the effects of iASPP depletion on cell viability, $1 \times 10^{6}$ Saos- 2 cells were plated and subsequently transfected with $10 \mu \mathrm{g}$ of each of the pRetroSUPER iASPP shRNA vectors or the pRetroSUPER scrambled vector. Forty-eight hours after washing, the cells were trypsinized and split into 150 -mm-diameter plates containing $600 \mu \mathrm{g} / \mathrm{ml} \mathrm{G} 418$. The plates were cultured for 4 weeks with twice-weekly medium changes containing G418. The plates were then washed with $10 \%$ methanol, and the colonies were visualized using a Giemsa stain according to the manufacturer's instructions (Sigma-Aldrich). pRetroSUPER-p73 was kindly provided by $\mathrm{K}$. Sabapathy (National Cancer Centre, Singapore). A corresponding plasmid containing a scrambled sequence was generated as outlined above.

Immunofluorescence. Saos- 2 cells were transiently transfected with pcDNA3, pcDNA3V5-iASPP, EGFP-N1, and/or 37AA. Twenty-four hours after transfection, cells were fixed in $4 \%$ paraformaldehyde and permeabilized with $0.3 \%$ Triton. Nuclei were stained with DAPI, and iASPP was detected using a rabbit V5 antibody (Invitrogen) and anti-rabbit Texas red secondary antibody (Molecular Probes).

Animals and xenograftstudies. Female CD-1 nude mice (mean initial weight, $25 \mathrm{~g}$ ) were housed in groups of 5 at $19^{\circ} \mathrm{C}$ to $23^{\circ} \mathrm{C}$ with a 12 -hour light/ 12-hour dark cycle. They had free access to food and water. Experimental work was carried out in accordance with UK Home Office regulations and approved by the local Ethical Review Panel at the University of Glasgow. Cell suspensions of $10^{6}$ exponentially growing cells were injected in each flank. Tumors (typically $6 \mathrm{~mm}$ in diameter) were palpable 6 days after subcutaneous injection. Gene therapy treatments were administered by intravenous tail vein injection on the day tumors became apparent (day 0 ) and on days 2, 4, 6, and 8 as previously described (36). These treatments consisted of nanoparticles of polypropylenimine dendrimer (generation 3, $\left.\mathrm{PPI}_{\mathrm{G} 3}, \mathrm{DAB}-\mathrm{Am} 16\right)$ and plasmid DNA (5:1 w/w, $50 \mu \mathrm{g}$ DNA) encoding either 37AA, tr105, or wtp53. Free $\mathrm{PPI}_{\mathrm{G} 3}$ dendrimer was also administered systemically. Control mice were injected with the plasmid DNAs or received no treatment. Animals $(n=6)$ were weighed daily, and their tumor volume (determined from caliper measurements and calculated as $d^{3} \times \pi / 6$, where $d$ represents diameter) was recorded.

\section{Acknowledgments}

We thank Bert Vogelstein, Tim Crook, Gerry Melino, John Reed, Kanaga Sabapathy, Andy Phillips, Ken Parkinson, and Ed Harlow for reagents and Paul Harrison, Gareth Inman, and Simon Wilkinson for critical reading of the manuscript. This work was supported by Cancer Research UK (K.M. Ryan) and the Biotechnology and Biological Sciences Research Council (A.G. Schätzlein). K.M. Ryan is a Cancer Research UK Senior Cancer Research Fellow.

Received for publication April 26, 2006, and accepted in revised form January 9, 2007.

Address correspondence to: Kevin M. Ryan, Beatson Institute for Cancer Research, Garscube Estate, Switchback Road, Glasgow G61 1BD, United Kingdom. Phone: 44-1413303655; Fax: 441419426521; E-mail: k.ryan@beatson.gla.ac.uk.

Helen S. Bell's present address is: Department of Biomedical Science, University of Aberdeen, Aberdeen, United Kingdom.

Daniele Bergamaschi's present address is: Centre for Cutaneous Research, Barts and the London, Queen Mary's School of Medicine and Dentistry, University of London, London, United Kingdom.

Christine Dufes's present address is: Strathclyde Institute of Pharmacy and Biomedical Sciences, University of Strathclyde, Glasgow, United Kingdom.

Andreas G. Schätzlein's present address is: School of Pharmacy, University of London, London, United Kingdom.
1. Vogelstein, B., Lane, D., and Levine, A.J. 2000. Surfing the p53 network. Nature. 408:307-310.

2. Crighton, D., and Ryan, K.M. 2004. Splicing DNAdamage responses to tumour cell death. Biochim. Biophys. Acta. 1705:3-15.

3. Vousden, K.H., and Lu, X. 2002. Live or let die: the cell's response to p53. Nat. Rev. Cancer. 2:594-604.

4. Lowe, S.W., Jacks, T., Housman, D.E., and Ruley, H.E. 1994. Abrogation of oncogene-associated apoptosis allows transformation of p53-deficient cells. Proc. Natl. Acad. Sci. U. S. A. 91:2026-2030.

5. Johnstone, R.W., Ruefli, A.A., and Lowe, S.W. 2002. Apoptosis: a link between cancer genetics and chemotherapy. Cell. 108:153-164.

6. Lane, D.P., and Lain, S. 2002. Therapeutic exploitation of the 53 pathway. Trends Mol. Med. 8:S38-S42.

7. Vassilev, L.T., et al. 2004. In vivo activation of the p53 pathway by small-molecule antagonists of MDM2. Science. 303:844-848.

8. Yang, Y., et al. 2005. Small molecule inhibitors of HDM2 ubiquitin ligase activity stabilize and activate p53 in cells. Cancer Cell. 7:547-559.

9. Soussi, T., and Lozano, G. 2005. p53 mutation heterogeneity in cancer. Biochem. Biophys. Res. Commun. 331:834-842.

10. Yang, A., Kaghad, M., Caput, D., and McKeon, F. 2002. On the shoulders of giants: $\mathrm{p} 63, \mathrm{p} 73$ and the rise of p53. Trends Genet. 18:90-95.

11. De Laurenzi, V., and Melino, G. 2000. Evolution of functions within the $\mathrm{p} 53 / \mathrm{p} 63 / \mathrm{p} 73$ family. Ann N.Y. Acad. Sci. 926:90-100.

12. Moll, U.M., Erster, S., and Zaika, A. 2001. p53, p63 and $\mathrm{p} 73$--solos, alliances and feuds among family members. Biochim. Biophys. Acta. 1552:47-59.
13. Irwin, M.S., et al. 2003. Chemosensitivity linked to p73 function. Cancer Cell. 3:403-410.

14. Benard, J., Douc-Rasy, S., and Ahomadegbe, J.C. 2003. TP53 family members and human cancers. Hum. Mutat. 21:182-191.

15. Haupt, Y., Rowan, S., Shaulian, E., Vousden, K.H., and Oren, M. 1995. Induction of apoptosis in HeLa cells by trans-activation-deficient p53. Genes Dev. 9:2170-2183

16. Bell, L.A., O'Prey, J., and Ryan, K.M. 2006. DNAbinding independent cell death from a minimal proapoptotic region of E2F-1. Oncogene. 25:5656-5663.

17. Cho, Y., Gorina, S., Jeffrey, P.D., and Pavletich, N.P. 1994. Crystal structure of a p53 tumor suppressorDNA complex: understanding tumorigenic mutations. Science. 265:346-355.

18. el-Deiry, W.S., et al. 1993. WAF1, a potential media- 
tor of $\mathrm{p} 53$ tumor suppression. Cell. 75:817-825.

19. Polyak, K., Xia, Y., Zweier, J.L., Kinzler, K.W., and Vogelstein, B. 1997. A model for p53-induced apoptosis. Nature. 389:300-305.

20. Miyashita, T., and Reed, J.C. 1995. Tumor suppressor $\mathrm{p} 53$ is a direct transcriptional activator of the human bax gene. Cell. 80:293-299.

21. Scheffner, M., Werness, B.A., Huibregtse, J.M., Levine, A.J., and Howley, P.M. 1990. The E6 oncoprotein encoded by human papillomavirus types 16 and 18 promotes the degradation of $\mathrm{p} 53$. Cell. 63:1129-1136.

22. Lowe, S.W., Ruley, H.E., Jacks, T., and Housman, D.E. 1993. p53-dependent apoptosis modulates the cytotoxicity of anticancer agents. Cell. 74:957-967

23. Slee, E.A., and Lu, X. 2003. The ASPP family: deciding between life and death after DNA damage. Toxicol. Lett. 139:81-87.

24. Bergamaschi, D., et al. 2004. ASPP1 and ASPP2: common activators of p53 family members. Mol. Cell. Biol. 24:1341-1350.

25. Samuels-Lev, Y., et al. 2001. ASPP proteins specifically stimulate the apoptotic function of p53. Mol. Cell. 8:781-794.

26. Thornton, J.K., et al. 2006. The tumour-suppressor protein ASPP1 is nuclear in human germ cells and can modulate ratios of CD44 exon V5 spliced isoforms in vivo. Oncogene. 25:3104-3112.

27. Kobayashi, S., et al. 2005. 53BP2 induces apoptosis through the mitochondrial death pathway. Genes Cells. 10:253-260.

28. Melino, G., De Laurenzi, V., and Vousden, K.H. 2002. p73: friend or foe in tumorigenesis. Nat. Rev. Cancer. 2:605-615.

29. Flores, E.R., et al. 2002. p63 and p73 are required for $\mathrm{p} 53$-dependent apoptosis in response to DNA damage. Nature. 416:560-564.

30. Toh, W.H., Kyo, S., and Sabapathy, K. 2005. Relief of p53-mediated telomerase suppression by $\mathrm{p} 73$. J. Biol. Chem. 280:17329-17338.

31. Nakano, K., and Vousden, K.H. 2001. PUMA, a novel proapoptotic gene, is induced by $\mathrm{p} 53 . \mathrm{Mol}$. Cell. 7:683-694.

32. Yu, J., Zhang, L., Hwang, P.M., Kinzler, K.W., and Vogelstein, B. 2001. PUMA induces the rapid apoptosis of colorectal cancer cells. Mol. Cell. 7:673-682.

33. Melino, G., et al. 2004. p73 induces apoptosis via PUMA transactivation and Bax mitochondrial translocation. J. Biol. Chem. 279:8076-8083.

34. Gonzalez, S., Prives, C., and Cordon-Cardo, C. 2003. p73alpha regulation by $\mathrm{Chk} 1$ in response to DNA damage. Mol. Cell. Biol. 23:8161-8171.

35. Wu, G.S., et al. 1997. KILLER/DR5 is a DNA damage-inducible p53-regulated death receptor gene. Nat. Genet. 17:141-143.

36. Dufes, C., et al. 2005. Synthetic anticancer gene medicine exploits intrinsic antitumor activity of cationic vector to cure established tumors. Cancer Res. 65:8079-8084.

37. Zinselmeyer, B.H., Mackay, S.P., Schatzlein, A.G., and Uchegbu, I.F. 2002. The lower-generation polypropylenimine dendrimers are effective genetransfer agents. Pharm. Res. 19:960-967.

38. Marin, M.C., et al. 1998. Viral oncoproteins discriminate between p53 and the p53 homolog p73. Mol. Cell. Biol. 18:6316-6324.

39. Bergamaschi, D., et al. 2003. iASPP oncoprotein is a key inhibitor of p53 conserved from worm to human. Nat. Genet. 33:162-167.

40. Zhang, X., Wang, M., Zhou, C., Chen, S., and Wang, J. 2005. The expression of iASPP in acute leukemias. Leuk. Res. 29:179-183.

41. Gong, J.G., et al. 1999. The tyrosine kinase c-Abl regulates p73 in apoptotic response to cisplatininduced DNA damage. Nature. 399:806-809.

42. Wang, W., Kim, S.H., and El-Deiry, W.S. 2006. Small-molecule modulators of p53 family signaling and antitumor effects in p53-deficient human colon tumor xenografts. Proc. Natl. Acad. Sci. U. S. A. 103:11003-11008.

43. Slee, E.A., et al. 2004. The N-terminus of a novel isoform of human iASPP is required for its cytoplasmic localization. Oncogene. 23:9007-9016.

44. Kinsella, T.M., and Nolan, G.P. 1996. Episomal vectors rapidly and stably produce high-titer recombinant retrovirus. Hum. Gene Ther. 7:1405-1413. 